Meta

Journal des tradlucteurs

Translators' Journal

\title{
Bulletin du Cercle des Traducteurs
}

\section{Paul A. Horguelin}

Volume 11, numéro 3, septembre 1966

URI : https://id.erudit.org/iderudit/010478ar

DOI : https://doi.org/10.7202/010478ar

Aller au sommaire du numéro

Éditeur(s)

Les Presses de l'Université de Montréal

\section{ISSN}

0026-0452 (imprimé)

1492-1421 (numérique)

Découvrir la revue

Citer ce compte rendu

Horguelin, P. A. (1966). Compte rendu de [Bulletin du Cercle des Traducteurs]. Meta, 11(3), 102-103. https://doi.org/10.7202/010478ar d'utilisation que vous pouvez consulter en ligne.

https://apropos.erudit.org/fr/usagers/politique-dutilisation/ 


\section{Bulletin \\ du Cercle des Traducteurs}

Depuis plusieurs années, le Cercle des Traducteurs (ex-Société des diplômés de l'Institut de Traduction) publie un bulletin semestriel à l'intention de ses membres. Que contient ce bulletin? Quelques brèves nouvelles sur l'activité du Cercle, une bibliographie d'ouvrages de traduction récemment parus et, principalement, des comptes rendus de séances d'étude. Ces derniers offrent pour les traducteurs un intérêt particulier; en effet, les animateurs des séances d'étude sont en général des chefs de services de traduction ou des traducteurs spécialisés. Les problèmes de traduction qu'ils soumettent, les solutions qu'ils proposent - et que les membres discutent - ont donc un but essentiellement pratique: trouver des équivalents à des termes qui présentent une réelle difficulté, soit qu'ils soient trop récents pour avoir retenu l'attention des lexicographes (notamment dans le domaine technique), soit qu'ils désignent une réalité typiquement canadienne et, de ce fait, nécessitent une solution canadienne. L'avantage de la formule est évident: l'animateur, en ne choisissant que des termes auxquels il s'est lui-même buté, élimine l'étude de problèmes ayant déjà reçu une solution; la discussion entre les membres qui forment pour ainsi dire un brain-trust - permet d'envisager toutes les facettes de la question et de bénéficier de nombreuses compétences; enfin, l'animateur motive généralement sa solution en expliquant la démarche suivie pour y parvenir: or, il est souvent aussi intéressant de savoir comment on est arrivé à une solution que de connaître la solution elle-même.

Le dernier bulletin du Cercle (IV, 1) porte sur la terminologie de l'automobile, de l'électricité et de la publicité. Voici un échantillon des nombreux équivalents intéressants qu'on y relève: Direct-acting shock absorber $=$ Amortisseur téleseopique (bel exemple de modulation !) ; Turn-key contract = Contrat $\ll$ clés en main $» ;$ Impulse sales $=$ Ventes de choe. Deux vocabulaires complètent les comptes rendus: l'un relatif à l'électrotechnique, l'autre, à l'aménagement hydro-électrique.

Les bulletins du Cercle des Traducteurs constituent une précieuse source de renseignements pour le traducteur, et il est regrettable qu'ils ne reçoivent pas une plus large diffusion. La même remarque s'applique d'ailleurs à de nombreuses publications de ce genre (bulletins, fiches, vocabulaires, etc.) provenant de divers organismes, entreprises commerciales et sociétés de tradueteurs. 
On a assisté dernièrement à une véritable éclosion de ces moyens d'information, qui répondent à un besoin réel. Malheureusement, tous ces efforts louables gardent, à notre avis, un caractère trop privé: seuls quelques privilégiés ont accès à ces sources de documentation. À l'heure où l'on parle de fédération et de fusion, où l'idée d'un fichier central est dans l'air à Québec et à Ottawa, la nécessité d'une coordination de toutes les bonnes volontés et compétences semble s'imposer. Il est temps, croyons-nous, que la recherche terminologique cesse d'être rigoureusement cloisonnée; il faut que l'ensemble de la cormmunauté des traducteurs puisse en bénéficier. Les ouvriers et les matériaux sont là, il ne manque que le grand architecte !

Paul A. Horguelin Montréal 